\title{
Lipid-Lowering Efficacy of Ezetimibe in Patients with Atherosclerotic Cardiovascular Disease: A Systematic Review and Meta-Analyses
}

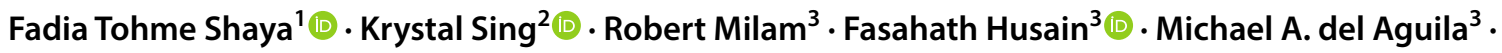 \\ Miraj Y. Patel ${ }^{4}$ (1)
}

Published online: 14 November 2019

(c) The Author(s) 2019

\begin{abstract}
Introduction Patients with atherosclerotic cardiovascular disease (ASCVD), especially those with recent ( $<1$ year) acute coronary syndrome (ACS), are at high risk for recurrent cardiovascular events. This risk can be reduced by lowering lowdensity lipoprotein cholesterol (LDL-C) levels. A comprehensive meta-analysis on the LDL-C-lowering efficacy of ezetimibe is lacking. This study attempts to address this gap.

Methods A systematic literature review of randomized controlled trials evaluating the LDL-C-lowering efficacy of ezetimibe in the ASCVD population was conducted. MEDLINE, EMBASE, and the Cochrane Central Register of Controlled Trials were searched for publications from database inception to August 2018 and for conference abstracts from 2015 to August 2018. Meta-analyses were conducted to evaluate the LDL-C-lowering efficacy of ezetimibe in the ASCVD population and the recent ACS subgroup.

Results In total, 12 studies were eligible for the meta-analyses. Treatment with combination ezetimibe plus statin therapy showed greater absolute LDL-C reduction than statin monotherapy (mean difference $-21.86 \mathrm{mg} / \mathrm{dL} ; 95 \%$ confidence interval $[\mathrm{CI}]-26.56$ to $-17.17 ; p<0.0001$ ) after 6 months of treatment (or at a timepoint closest to 6 months). Similarly, in patients with recent ACS, combination ezetimibe plus statin therapy was favorable compared with statin monotherapy (mean treatment difference $-19.19 \mathrm{mg} / \mathrm{dL} ; 95 \% \mathrm{CI}-25.22$ to -13.16 ; $p<0.0001$ ).

Conclusions Ezetimibe, when added to statin therapy, provided a modest additional reduction in LDL-C compared with statin monotherapy. However, this may not be sufficient for some patients with ASCVD who have especially high LDL-C levels despite optimal statin therapy.
\end{abstract}

\section{Introduction}

Patients with atherosclerotic cardiovascular disease (ASCVD) are at high risk of cardiovascular events [1], and those with a recent history of a cardiovascular event (within

Electronic supplementary material The online version of this article (https://doi.org/10.1007/s40256-019-00379-9) contains supplementary material, which is available to authorized users.

Fadia Tohme Shaya

fshaya@rx.umaryland.edu

1 Pharmaceutical Health Services Research, University of Maryland School of Pharmacy, 220 Arch Street, 12th Floor, Room 01-204, Baltimore, MD 21201, USA

2 Regeneron Pharmaceuticals, Inc., Tarrytown, NY, USA

3 Doctor Evidence, Santa Monica, CA, USA

4 Sanofi, Bridgewater, NJ, USA the previous 12 months) are at even greater risk of a recurrent event [2-6]. Patients with clinical ASCVD are typically defined as those with acute coronary syndrome (ACS) or history of myocardial infarction, stable or unstable angina, coronary revascularization, ischemic stroke, transient ischemic attack, or peripheral arterial disease [7]. Reduction of lowdensity lipoprotein cholesterol (LDL-C) is recommended to prevent future cardiovascular events.

The 2018 multi-society guideline on the management of blood cholesterol recommended the use of maximally tolerated statins for LDL-C reduction in patients with ASCVD [8]. However, even with statin therapy, many patients with clinical ASCVD will need additional lipid-lowering therapies (LLTs) to achieve LDL-C levels $<70 \mathrm{mg} / \mathrm{dL}$ [9]. In fact, for those with ASCVD at very high risk for future events, the guidelines recommended additional LDL-C-lowering therapies (ezetimibe and/or proprotein convertase subtilisin/kexin 


\section{Key Points}

The meta-analyses demonstrated that, for patients with atherosclerotic cardiovascular disease (ASCVD), ezetimibe added to statin therapy provided an additional reduction of $22 \mathrm{mg} / \mathrm{dL}$ in low-density lipoprotein cholesterol (LDL-C) compared with statin monotherapy.

LDL-C $>70 \mathrm{mg} / \mathrm{dL}$ is considered a threshold at which additional LDL-C-lowering therapy may be needed in patients with ASCVD and at very high risk of recurrent events; the magnitude of the incremental reduction in LDL-C with ezetimibe observed here may not be sufficient for many patients.

type 9 [PCSK9] inhibitors) if LDL-C is $>70 \mathrm{mg} / \mathrm{dL}$ with maximally tolerated statins.

Ezetimibe is a cholesterol absorption inhibitor that has been shown to provide a $17-23 \%$ further reduction in LDL-C levels when added to statin therapy [10-12] and also a reduction of cardiovascular events in patients with ACS in IMPROVE-IT (Improved Reduction of Outcomes: Vytorin Efficacy International Trial) [12]. Patients with ACS are particularly at risk of recurrent cardiovascular events, with approximately $20 \%$ recurrence in the first year, as demonstrated by an analysis of clinical registry data [4].

Although results of individual trials with ezetimibe are available, a comprehensive appraisal of the literature on the LDL-C-lowering efficacy of ezetimibe since IMPROVE-IT is lacking. There is also no review focusing on studies of the recent ACS population. In an effort to address these gaps, we conducted a systematic literature review and meta-analyses to assess the LDL-C-lowering efficacy of ezetimibe in patients with ASCVD and the high-risk subgroup of patients with recent (event in the last 1 year) ACS.

\section{Methods}

We conducted a systematic literature review to find randomized controlled trials (RCTs) that measured the impact of ezetimibe on lowering LDL-C. Meta-analyses were then conducted to assess LDL-C lowering among patients with ASCVD and/or ACS. Sensitivity analyses were conducted to assess the impact of analytic decisions on the primary results.

\subsection{Literature Sources}

We searched three electronic bibliographic databases: MEDLINE (via the PubMed platform), Embase (via OVID), and the Cochrane Central Database of Controlled Trials (via the Wiley platform). The databases were searched for all publications from starting date through to 27 August 2018 for MEDLINE and to 28 August 2018 for Embase and the Cochrane Central Database of Controlled Trials. A manual search was also conducted on the reference lists of identified eligible studies and published systematic literature reviews (using the same timeframe as the literature searches for eligible RCTs). In addition, we searched conference abstracts listed in Embase and manually searched specific congress proceedings (a complete list of the congress proceedings searched is provided in the Electronic Supplementary Material [ESM]). Congresses were searched from January 2015 through August 2018, inclusive. The search criteria for each database are provided in Tables S1-S3 in the ESM.

\subsection{Eligibility Criteria}

Study eligibility was guided by methods published by The Cochrane Collaboration [13]. RCTs, of any setting, reported in English, and reporting the population, intervention, comparison, and outcomes of interest (as described below) were eligible for inclusion if they had a follow-up of $\geq 30$ days and reported LDL-C outcomes at $\geq 30$ days following initiation of treatment. Studies were excluded if they did not meet these eligibility criteria; we also excluded duplicate publications, in vitro studies, animal studies, and studies not reporting LDL-C outcomes by ezetimibe initiation status.

The target population consisted of patients with ASCVD, defined as those with a prior history of myocardial infarction, stable or unstable angina, coronary or other arterial revascularization, stroke, transient ischemic attack, or peripheral arterial disease. The LDL-C-lowering intervention assessed was ezetimibe therapy, received with or without other LLTs. Primary efficacy outcomes of interest included the mean change in LDL-C from baseline at 6 months, with sensitivity analyses at 1-, 3-, 9-, and 12-month timepoints (or closest reported timepoint to those).

\subsection{Study Selection}

Two reviewers independently screened the identified literature, based on the abovementioned eligibility criteria, in a two-step process: (1) title/abstract screening and (2) fulltext screening. If concordance between the two reviewers was below $80 \%$ for the title/abstract screen, then the entire bibliography was to be re-screened; however, a re-screen was unnecessary as concordance was $94.6 \%$. After removing duplicates, a full-text screen was performed on the references identified from the title/abstract screening. Reasons provided for inclusion/exclusion of studies at both stages were documented during screening. Any discrepancies that occurred during title/abstract screening or full-text screening were identified and resolved by a third independent reviewer. 


\subsection{Data Extraction}

Data were extracted by two analysts utilizing the $\mathrm{DOC}^{\mathrm{TM}}$ DATA software platform (version 2.0, Doctor Evidence, LLC, Santa Monica, CA, USA), and any discrepancy was resolved by a third independent reviewer. Data and metadata were obtained from the text manually, and digitizer software (Grab-It $\left.{ }^{\mathrm{TM}}\right)$ was used to capture relevant data points from figures, charts, and tables. Final quality control auditing was performed to ensure the accuracy and consistency of data across the dataset, including the sampling of $25 \%$ of all extracted data for quality assurance. A study quality appraisal was conducted using the Cochrane Collaboration Risk of Bias Assessment Tool for systematic reviews [13] to evaluate seven domains of bias: random sequence generation (selection bias), allocation concealment (selection bias), blinding of participants and personnel (performance bias), blinding of outcome assessment (detection bias), incomplete outcome data (attrition bias), selective reporting (reporting bias), and other sources of bias (other bias).

\subsection{Data Synthesis and Analysis}

Direct (pair-wise) meta-analyses were conducted comparing ezetimibe therapy plus background LLT versus the same background LLT (which could have been no background LLT); all analyses were performed using the "metafor" package in R software version 3.5.1 [14]. Analyses of overall estimates of effect sizes used the random-effects model of DerSimonian and Laird [13]. Heterogeneity of effect size was assessed with the $Q$ test statistic [13], using cut-off values of $25 \%, 50 \%$, and $75 \%$ to assign low, moderate, and high degrees of heterogeneity, respectively. Comparisons of heterogeneity between meta-analyses were performed based on the $I^{2}$ statistic [13].

The primary analysis compared the mean LDL-C change from baseline between combination ezetimibe plus background LLT versus background LLT comparator for all studies at 6 months or at the reported timepoint closest to 6 months. Sensitivity analyses included the assessment of the LDL-C change from baseline over shorter (1-3 months) or longer (9-12 months) durations. A sensitivity analysis with the largest trial removed was also conducted because of its disproportionately high weight in the primary analysis. Separate analyses for trials enrolling patients who were statin naïve versus those with a history of statin use were also conducted. In addition, analyses were performed on the subgroup of trials that only enrolled patients with recent ( $<1$ year) ACS.

\section{Results}

\subsection{Studies Identified Through the Systematic Literature Review}

The systematic review flow diagram is shown in Fig. 1. A total of 14 records matching the eligibility criteria were identified through the systematic literature review (refer to Tables S4 and S5 in the ESM for a summary of studies included). Of these, 12 records reported the primary outcome of either LDL-C change from baseline or LDL-C level at the endpoint, or both, and had adequate independent information available that could be used for the meta-analysis. The justifications recorded for the inclusion/exclusion of the identified records are summarized in Tables S4 and S5 in the ESM; the resulting 12 studies included in the meta-analysis are listed in Table 1, along with study design, treatment arms, duration, baseline demographic characteristics, and baseline LDL-C values for each study.

Although the objective was to assess LDL-C lowering with ezetimibe therapy received with or without other LLTs compared with the same background LLTs, all included studies compared combination ezetimibe plus statin therapy with statin monotherapy. Across the studies, the mean age of participants ranged from 57 to 71 years according to treatment group (Table 1). A key difference between the 12 studies was the definition of cardiovascular history used as an inclusion criterion, as summarized in Table S6 in the ESM. The primary LDL-C outcomes of interest reported in the included studies are summarized in Table S7 in the ESM.

Across the 12 trials, the risk of selection bias was mainly unclear for random sequence generation and allocation concealment, as authors did not describe the randomization methodology in sufficient detail. The risk of selection bias was mainly low for random sequence generation and unclear for allocation concealment as authors did not describe the randomization methodology in enough detail. The risk of performance bias, pertaining to blinding of participants and personnel, was assessed as high among four trials, low among two trials, and unclear among six trials. The risk of detection bias, pertaining to blinding of outcome assessment, was rated as high for one trial. The risk of attrition bias (pertaining to incomplete outcome data) and the risk of reporting bias (pertaining to selective reporting of outcomes) was predominantly low across all trials. The risk of other sources of bias was predominantly unclear across all trials. A summary of the risk of bias across the 12 trials is provided in Table S8 in the ESM. 


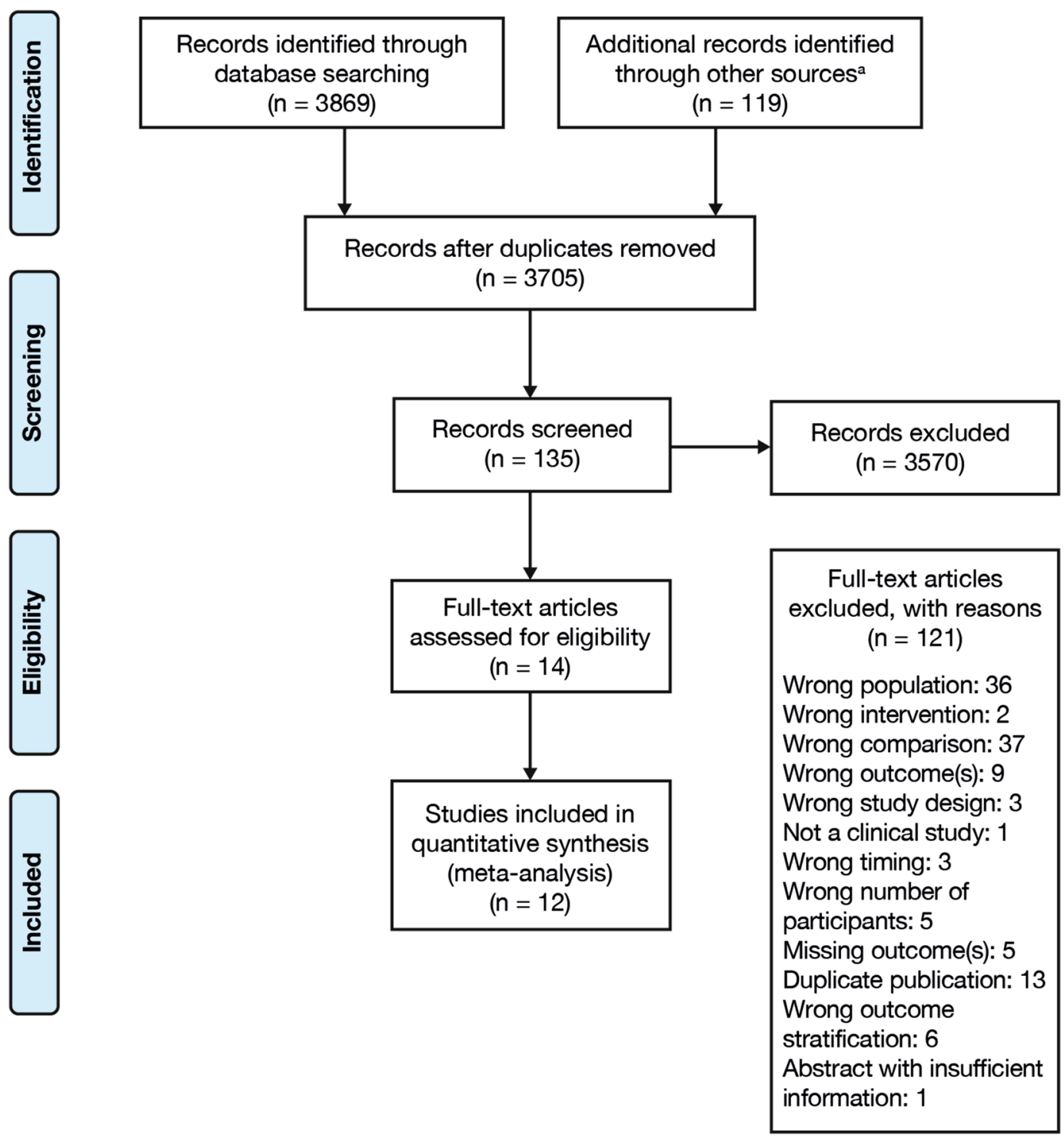

Fig. 1 Study flow diagram of the systematic review. Overall, 14 records were identified through the systematic literature review that reported on patients with ASCVD, that assessed ezetimibe therapy (received with or without other lipid-lowering therapies), and reported LDL-C change, LDL-C at endpoint, or both. Of these, 12 records contained adequate and independent information sufficient for

\subsection{Efficacy of Low-Density Lipoprotein Cholesterol Lowering of Ezetimibe}

A forest plot showing the primary outcome of interest, LDL-C change from baseline, with combination ezetimibe plus statin therapy versus statin monotherapy, is presented in Fig. 2 for the main analysis of data collected at 6 months (or at the reported timepoint closest to 6 months). Overall, patients receiving combination ezetimibe were likely to experience an additional decrease in LDL-C $(-21.86 \mathrm{mg} /$ $\mathrm{dL} ; 95 \%$ confidence interval $[\mathrm{CI}]-26.56$ to -17.17 ; meta-analysis, including nine reporting on two-arm trials and three reporting on multi-arm trials. ${ }^{\mathrm{a}} 115$ records were manually entered, and four additional records were cited in protocols. ASCVD atherosclerotic cardiovascular disease, $L D L-C$ low-density lipoprotein cholesterol

$p<0.0001)$ compared with those receiving statin monotherapy. Sensitivity analyses of trials based on time of reporting of LDL-C change showed similar results for both shorter (1-3 months), at 6 months, and longer (9-12 months) treatment durations (overall LDL-C mean difference $-21.49 \mathrm{mg}$ / $\mathrm{dL} ; 95 \% \mathrm{CI}-28.45$ to $-14.53, p<0.0001$ at 1 month; $-19.96 \mathrm{mg} / \mathrm{dL} ; 95 \% \mathrm{CI}-28.07$ to $-11.85, p<0.0001$ at 3 months; $-20.75 \mathrm{mg} / \mathrm{dL} ; 95 \% \mathrm{CI}-26.92$ to -14.58 , $p<0.0001$ at 6 months; and $-19.36 \mathrm{mg} / \mathrm{dL} ; 95 \% \mathrm{CI}-24.42$ to $-14.30, p<0.0001$ at $9-12$ months; Figs. S1, S2, S3, and $\mathrm{S} 4$, respectively [see the ESM]). A sensitivity analysis 


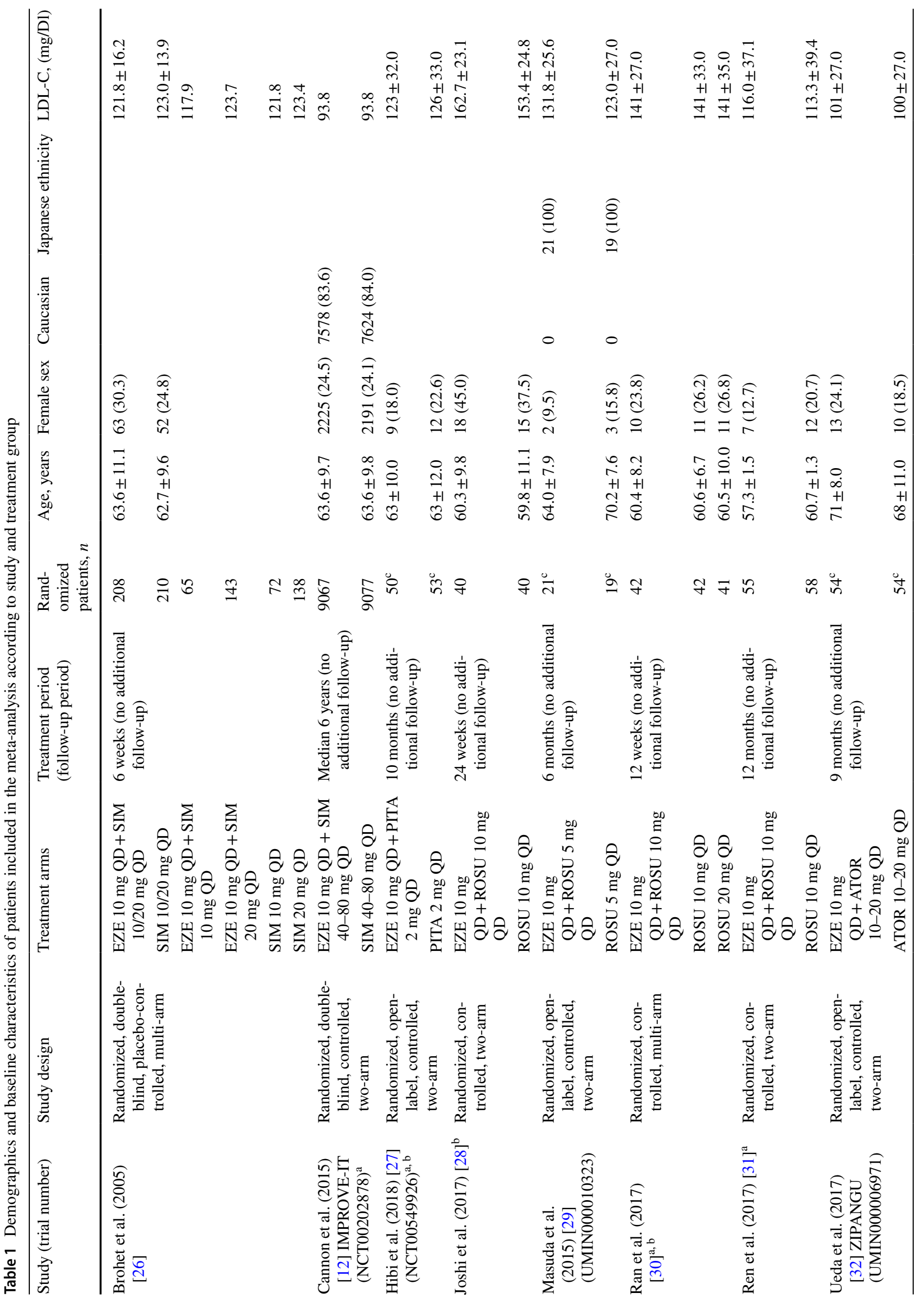




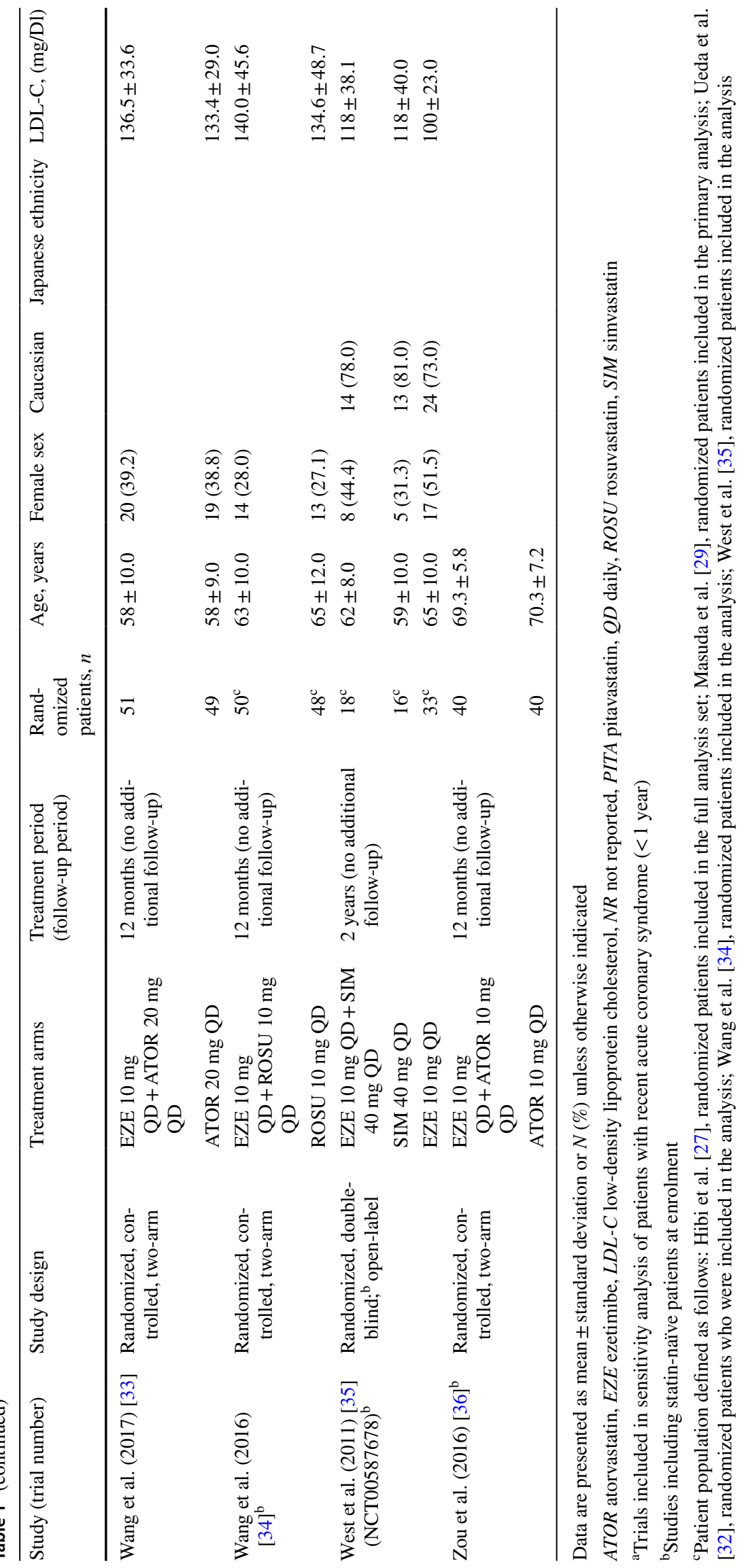




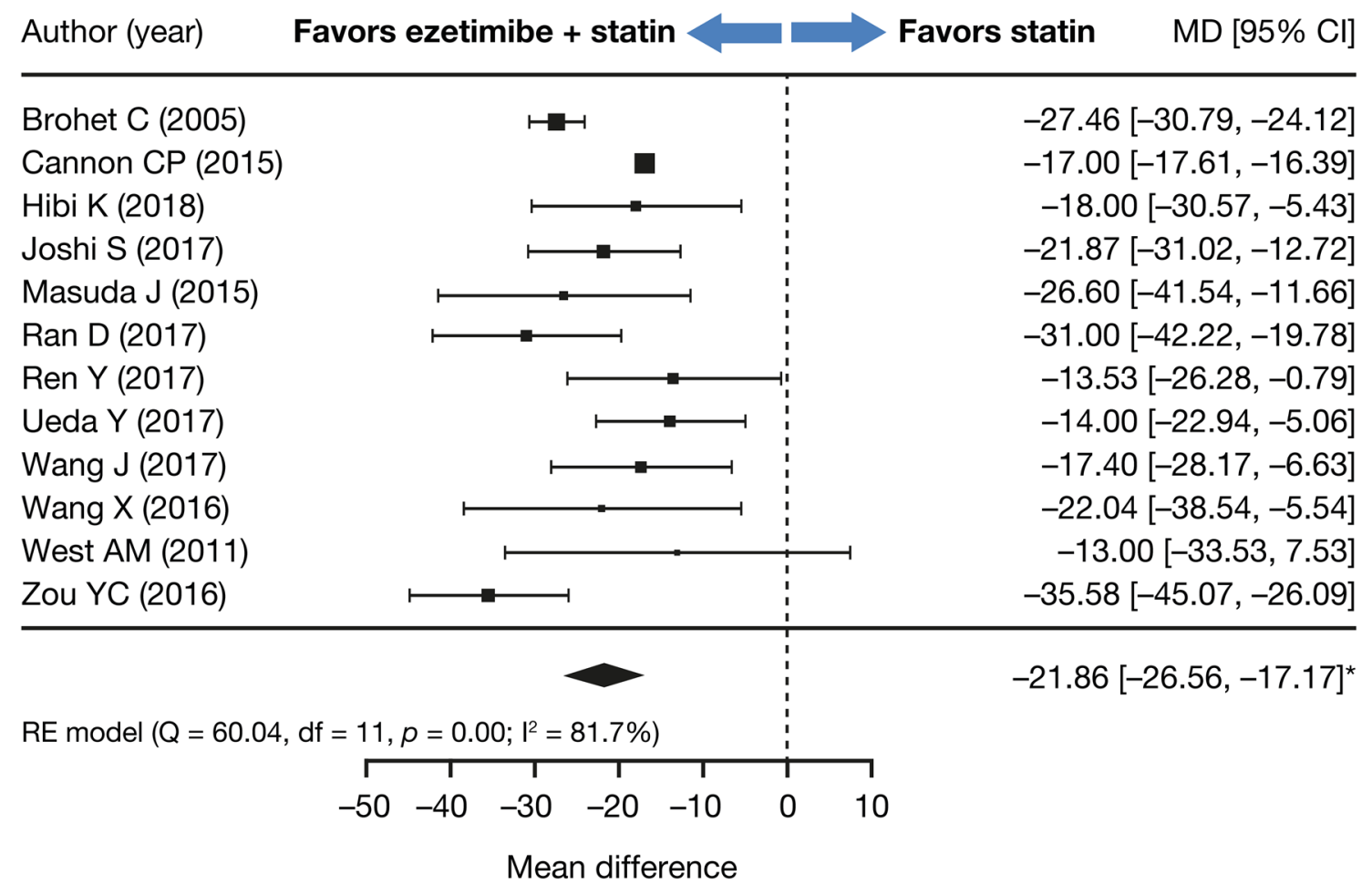

Fig. 2 Treatment difference in mean LDL-C change (mg/dL) from baseline between combination ezetimibe plus statin therapy and statin monotherapy comparator at 6 months or at the reported timepoint

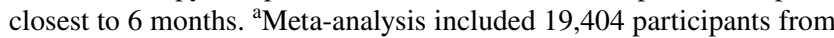

removing the study by Cannon et al. [12] yielded a similar result (LDL-C mean difference $-22.84 \mathrm{mg} / \mathrm{dL} ; 95 \% \mathrm{CI}$ -27.40 to $-18.28, p<0.0001$ at 6 months or at the reported timepoint closest to 6 months).

Analyses of studies according to whether patients were statin naïve or had a history of statin therapy at enrollment showed that patients receiving combination ezetimibe plus statin therapy had greater LDL-C reduction than those receiving statin monotherapy, irrespective of prior history of statin therapy (mean difference $-25.07 \mathrm{mg} / \mathrm{dL} ; 95 \%$ CI -31.73 to -18.41 and $-19.54 \mathrm{mg} / \mathrm{dL} ; 95 \% \mathrm{CI}-25.56$ to - 13.53, respectively; Fig. S5 in the ESM). There was no significant difference between the two groups $(p=0.2462)$.

\subsection{Subgroup Analysis of Patients with a History of Recent Acute Coronary Syndrome}

Similar to the results in overall population, analyses of the subpopulation with recent ( $<1$ year) ACS showed that patients receiving combination ezetimibe plus statin were more likely to experience a decrease in LDL-C levels than those receiving statin monotherapy (LDL-C mean difference of $-19.19 \mathrm{mg} / \mathrm{dL} ; 95 \% \mathrm{CI}-25.22$ to $-13.16, p<0.0001$; Fig. 3).
12 studies, who received treatment (ezetimibe + statin vs. statin) for a mean duration of 11.56 months. * $p<0.0001$. CI confidence interval, $L D L-C$ low-density lipoprotein cholesterol, $M D$ mean difference, $R E$ random effects

\section{Discussion}

Although previous studies have assessed the efficacy and safety of ezetimibe [15-20], this study is the first systematic literature review and meta-analysis of LDL-C lowering with ezetimibe in patients with ASCVD since the results of the IMPROVE-IT cardiovascular outcomes trial were published. In the present analysis, combination ezetimibe plus statin therapy resulted in a modest (relative to other treatment options) additional decrease in LDL-C compared with statin monotherapy (LDL-C mean difference $-21.86 \mathrm{mg} / \mathrm{dL}$ after 6 months of treatment), which was unaffected by treatment duration or a patient's prior history of statin therapy. Results were similar for patients with a recent ACS event (LDL-C mean difference of $-19.19 \mathrm{mg} / \mathrm{dL}$ ). These results are consistent with the 19-23\% LDL-C reduction reported previously for ezetimibe when added to statin therapy $[10,11]$.

The dose-dependent association between absolute LDL-C level and cardiovascular risk has been consistently demonstrated across statin trials. In the CTTC (Cholesterol Treatment Trialists Collaboration) study, a reduction of approximately $22 \%$ in major vascular events occurred per $1 \mathrm{mmol} / \mathrm{L}$ $(38.7 \mathrm{mg} / \mathrm{dL})$ lowering of LDL-C level. The incremental reduction in LDL-C with ezetimibe when added to statin 
Fig. 3 Subgroup analysis: LDL-C change $(\mathrm{mg} / \mathrm{dL})$ from baseline at 6 months, or at the reported timepoint closest to 6 months, limited to studies including patients with recent acute ( $<1$ year) coronary syndrome (Meta-analysis included 18,436 participants from four studies, who received treatment [ezetimibe plus statin vs. statin] for a mean duration of 11.90 months). $* p<0.0001$. $C I$ confidence interval, $L D L-C$ low-density lipoprotein cholesterol, $M D$ mean difference, $R E$ random effects

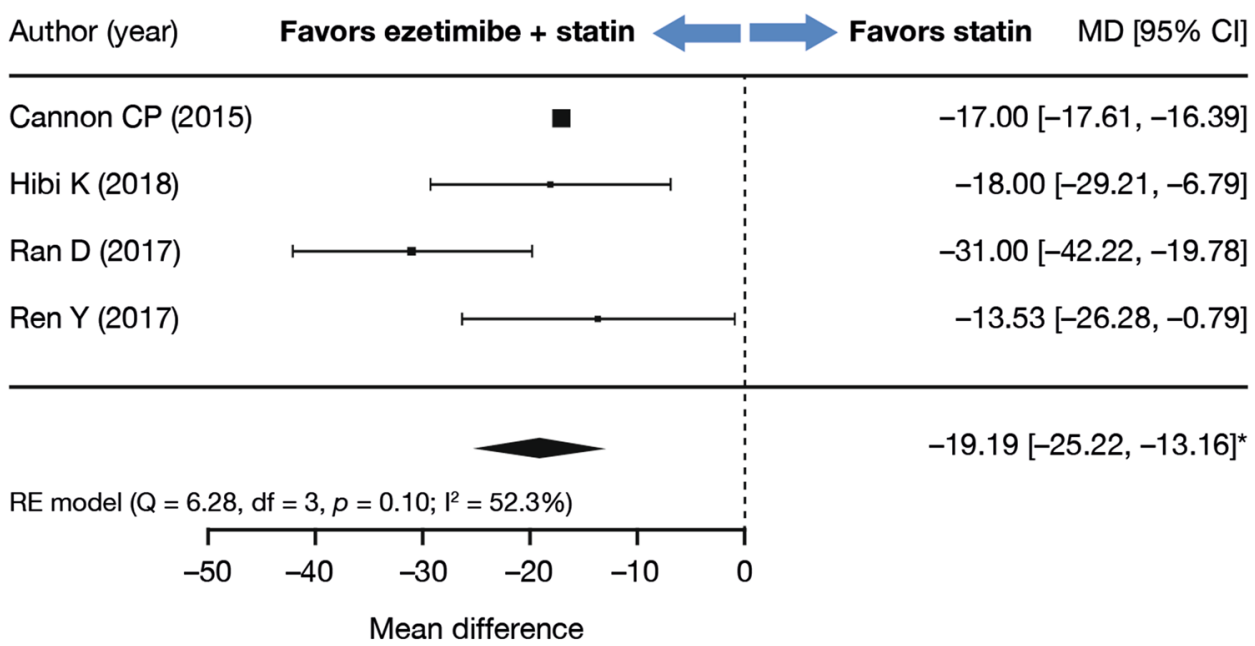

therapy may not be sufficient for patients at very high risk of cardiovascular events and with LDL-C levels more than 20-25 mg/dL from the desired threshold despite optimal statin use. Furthermore, the relatively low variability in patient response to ezetimibe observed in these analyses suggests that it may not be enough for those with very high LDL-C levels. In such patients, there may be a need to consider other non-statin LLTs, including PCSK9 inhibitors [21]. A 2019 National Lipid Association statement identified specific patient subgroups for whom it may be optimal to directly initiate a PCSK9 inhibitor based on potentially insufficient LDL-C and risk reduction on ezetimibe alone [22].

Similar considerations may also be important in patients with recent ACS because of the high risk for subsequent events in the months following an event [12, 23]. LDL-C reduction results from the analysis of the recent ACS population reported here were consistent with the IMPROVEIT cardiovascular outcomes trial [12] as well as the primary analysis reported here (general ASCVD population). The results remained similar in a sensitivity analysis with IMPROVE-IT removed.

Overall, results were also similar to those of other past studies. LDL-C lowering was consistent with that reported in previous studies including RCTs that did not meet our inclusion criteria and pooled analyses $[11,24]$. The low variability of LDL-C lowering with the addition of ezetimibe to statin therapy is in line with a previous report by Descamps et al. [25].

A limitation of the present analysis is that differences in clinical attributes of studied populations may introduce unmeasurable biases (participant cardiovascular inclusion criteria for each study are summarized in Table S6 in the ESM). There may also be potential publication bias in terms of which studies may have been published, but there is no clear way to quantify these potential biases. On the other hand, access to multiple clinical studies permits examination from a broader evidence base than provided through the analysis of a single clinical trial. A patient-level meta-analysis would complement these results, although gaining access to such data would be challenging.

\section{Conclusions}

The evidence from this systematic literature review and meta-analyses showed that patients with ASCVD receiving combination ezetimibe plus statin therapy experienced an additional $22 \mathrm{mg} / \mathrm{dL}$ reduction in LDL-C compared with patients receiving statin therapy alone. The modest incremental reduction in LDL-C suggests that patients with ASCVD and LDL-C levels more than 20-25 mg/dL from the desired threshold despite statin therapy may need other LLT options such as PCSK9 inhibitors.

Acknowledgements The following people from the study sponsors reviewed and provided editorial comments on the manuscript: Robert Sanchez, PhD, Yingxin $\mathrm{Xu}, \mathrm{PhD}$, and Serban Iorga, PhD (all Regeneron Pharmaceuticals, Inc.). Medical writing assistance and editorial support, under the direction of the authors, was provided by Rachel Dunn, PhD, of Prime (Knutsford, UK) funded by Sanofi and Regeneron Pharmaceuticals, Inc., according to Good Publication Practice guidelines. The sponsors were involved in the study design, collection, analysis, and interpretation of data as well as data checking of information provided in the manuscript. The authors had unrestricted access to study data, were responsible for all content and editorial decisions, and received no honoraria related to the development of this publication.

\section{Compliance with Ethical Standards}

Funding This analysis was funded by Sanofi and Regeneron Pharmaceuticals, Inc.

Conflict of interest Fadia T. Shaya is Professor at the University of Maryland School of Pharmacy and has received consulting fees from Sanofi/Regeneron for general advice on study design. Krystal Sing is 
an employee of and stockholder in Regeneron Pharmaceuticals, Inc. Robert Milam, Fasahath Husain, and Michael A. del Aguila are employees of Doctor Evidence, which was contracted by Sanofi/Regeneron to participate in the design, conduct, and reporting of this study. Miraj Y. Patel is an employee of and stockholder in Sanofi.

Open Access This article is distributed under the terms of the Creative Commons Attribution-NonCommercial 4.0 International License (http://creativecommons.org/licenses/by-nc/4.0/), which permits any noncommercial use, distribution, and reproduction in any medium, provided you give appropriate credit to the original author(s) and the source, provide a link to the Creative Commons license, and indicate if changes were made.

\section{References}

1. Piepoli MF, Hoes AW, Agewall S, Albus C, Brotons C, Catapano $\mathrm{AL}$, et al. 2016 European Guidelines on cardiovascular disease prevention in clinical practice: the Sixth Joint Task Force of the European Society of Cardiology and Other Societies on Cardiovascular Disease Prevention in Clinical Practice (constituted by representatives of 10 societies and by invited experts) developed with the special contribution of the European Association for Cardiovascular Prevention \& Rehabilitation (EACPR). Eur Heart J. 2016;37(29):2315-81.

2. Sanchis-Gomar F, Perez-Quilis C, Leischik R, Lucia A. Epidemiology of coronary heart disease and acute coronary syndrome. Ann Transl Med. 2016;4(13):256.

3. Hess CN, Clare RM, Neely ML, Tricoci P, Mahaffey KW, James SK, et al. Differential occurrence, profile, and impact of first recurrent cardiovascular events after an acute coronary syndrome. Am Heart J. 2017;187:194-203.

4. Jernberg T, Hasvold P, Henriksson M, Hjelm H, Thuresson M, Janzon M. Cardiovascular risk in post-myocardial infarction patients: nationwide real world data demonstrate the importance of a long-term perspective. Eur Heart J. 2015;36(19):1163-70.

5. Catapano AL, Graham I, De Backer G, Wiklund O, Chapman MJ, Drexel H, et al. 2016 ESC/EAS guidelines for the management of dyslipidaemias. Eur Heart J. 2016;37(39):2999-3058.

6. Motivala AA, Tamhane U, Ramanath VS, Saab F, Montgomery DG, Fang J, et al. A prior myocardial infarction: how does it affect management and outcomes in recurrent acute coronary syndromes? Clin Cardiol. 2008;31(12):590-6.

7. Lloyd-Jones DM, Morris PB, Ballantyne CM, Birtcher KK, Daly DD Jr, Writing Committee, et al. 2016 ACC Expert Consensus Decision Pathway on the role of non-statin therapies for LDL-cholesterol lowering in the management of atherosclerotic cardiovascular disease risk: a report of the American College of Cardiology Task Force on Clinical Expert Consensus Documents. J Am Coll Cardiol. 2016;68(1):92-125.

8. Grundy SM, Stone NJ, Bailey AL, Beam C, Birtcher KK, Blumenthal RS, et al. 2018 AHA/ACC/AACVPR/AAPA/ABC/ACPM/ ADA/AGS/APhA/ASPC/NLA/PCNA Guideline on the Management of Blood Cholesterol: A Report of the American College of Cardiology/American Heart Association Task Force on Clinical Practice Guidelines. Circulation. 2019;139(25):e1082-143.

9. Wong ND, Young D, Zhao Y, Nguyen H, Caballes J, Khan I, et al. Prevalence of the American College of Cardiology/American Heart Association statin eligibility groups, statin use, and low-density lipoprotein cholesterol control in US adults using the National Health and Nutrition Examination Survey 2011-2012. J Clin Lipidol. 2016;10(5):1109-18.
10. Catapano A, Toth PP, Tomassini JE, Tershakovec AM. The efficacy and safety of ezetimibe coadministered with statin therapy in various patient groups. Clin Lipidol. 2013;8(1):13-41.

11. Morrone D, Weintraub WS, Toth PP, Hanson ME, Lowe RS, Lin $\mathrm{J}$, et al. Lipid-altering efficacy of ezetimibe plus statin and statin monotherapy and identification of factors associated with treatment response: a pooled analysis of over 21,000 subjects from 27 clinical trials. Atherosclerosis. 2012;223(2):251-61.

12. Cannon CP, Blazing MA, Giugliano RP, McCagg A, White JA, Theroux $\mathrm{P}$, et al. Ezetimibe added to statin therapy after acute coronary syndromes. N Engl J Med. 2015;372(25):2387-97.

13. Cochrane Handbook for Systematic Reviews of Interventions. 2011 [Updated March 2011]. http://handbook.cochrane.org/. Accessed Apr 2018.

14. Viechtbauer W. Conducting meta-analyses in $\mathrm{R}$ with the metafor package. J Stat Soft. 2010;36(3):1-48.

15. Savarese G, De Ferrari GM, Rosano GM, Perrone-Filardi P. Safety and efficacy of ezetimibe: a meta-analysis. Int J Cardiol. 2015;201:247-52.

16. Fei Y, Guyatt GH, Alexander PE, El Dib R, Siemieniuk RAC, Vandvik PO, et al. Addition of ezetimibe to statins for patients at high cardiovascular risk: systematic review of patient-important outcomes. J Eval Clin Pract. 2018;24(1):222-31.

17. Battaggia A, Donzelli A, Font M, Molteni D, Galvano A. Clinical efficacy and safety of ezetimibe on major cardiovascular endpoints: systematic review and meta-analysis of randomized controlled trials. PLoS One. 2015;10(4):e0124587.

18. Mikhailidis DP, Lawson RW, McCormick AL, Sibbring GC, Tershakovec AM, Davies GM, et al. Comparative efficacy of the addition of ezetimibe to statin vs statin titration in patients with hypercholesterolaemia: systematic review and meta-analysis. Curr Med Res Opin. 2011;27(6):1191-210.

19. Vavlukis M, Vavlukis A. Adding ezetimibe to statin therapy: latest evidence and clinical implications. Drugs Context. 2018;7:212534.

20. Ai C, Zhang S, He Q, Shi J. Comparing the combination therapy of ezetimibe and atorvastatin with atorvastatin monotherapy for regulating blood lipids: a systematic review and meta-analyse. Lipids Health Dis. 2018;17(1):239.

21. Landmesser U, Chapman MJ, Farnier M, Gencer B, Gielen S, Hovingh GK, et al. European Society of Cardiology/European Atherosclerosis Society Task Force consensus statement on proprotein convertase subtilisin/kexin type 9 inhibitors: practical guidance for use in patients at very high cardiovascular risk. Eur Heart J. 2017;38(29):2245-55.

22. Robinson JG, Jayanna MB, Brown AS, Aspry K, Orringer C, Gill EA, et al. Enhancing the value of PCSK9 monoclonal antibodies by identifying patients most likely to benefit. J Clin Lipidol. 2019.

23. Schwartz GG, Steg PG, Szarek M, Bhatt DL, Bittner VA, Diaz R, et al. Alirocumab and cardiovascular outcomes after acute coronary syndrome. N Engl J Med. 2018;379(22):2097-107.

24. Hong SJ, Jeong HS, Ahn JC, Cha DH, Won KH, Kim W, et al. A phase III, multicenter, randomized, double-blind, active comparator clinical trial to compare the efficacy and safety of combination therapy with ezetimibe and rosuvastatin versus rosuvastatin monotherapy in patients with hypercholesterolemia: I-ROSETTE (Ildong Rosuvastatin \& Ezetimibe for Hypercholesterolemia) randomized controlled trial. Clin Ther. 2018;40(2):226-41.

25. Descamps O, Tomassini JE, Lin J, Polis AB, Shah A, Brudi P, et al. Variability of the LDL-C lowering response to ezetimibe and ezetimibe + statin therapy in hypercholesterolemic patients. Atherosclerosis. 2015;240(2):482-9.

26. Brohet C, Banai S, Alings AM, Massaad R, Davies MJ, Allen C. LDL-C goal attainment with the addition of ezetimibe to ongoing simvastatin treatment in coronary heart disease patients with hypercholesterolemia. Curr Med Res Opin. 2005;21(4):571-8. 
27. Hibi K, Sonoda S, Kawasaki M, Otsuji Y, Murohara T, Ishii $\mathrm{H}$, et al. Effects of ezetimibe-statin combination therapy on coronary atherosclerosis in acute coronary syndrome. Circ J. 2018;82(3):757-66.

28. Joshi S, Sharma R, Rao HK, Narang U, Gupta N. Efficacy of combination therapy of rosuvastatin and ezetimibe vs rosuvastatin monotherapy on lipid profile of patients with coronary artery disease. J Clin Diagn Res. 2017;11(12):OC28-31.

29. Masuda J, Tanigawa T, Yamada T, Nishimura Y, Sasou T, Nakata $\mathrm{T}$, et al. Effect of combination therapy of ezetimibe and rosuvastatin on regression of coronary atherosclerosis in patients with coronary artery disease. Int Heart J. 2015;56(3):278-85.

30. Ran D, Nie HJ, Gao YL, Deng SB, Du JL, Liu YJ, et al. A randomized, controlled comparison of different intensive lipid-lowering therapies in Chinese patients with non-ST-elevation acute coronary syndrome (NSTE-ACS): ezetimibe and rosuvastatin versus high-dose rosuvastatin. Int J Cardiol. 2017;235:49-55.

31. Ren Y, Zhu H, Fan Z, Gao Y, Tian N. Comparison of the effect of rosuvastatin versus rosuvastatin/ezetimibe on markers of inflammation in patients with acute myocardial infarction. Exp Ther Med. 2017;14(5):4942-50.
32. Ueda Y, Hiro T, Hirayama A, Komatsu S, Matsuoka H, Takayama $\mathrm{T}$, et al. Effect of ezetimibe on stabilization and regression of intracoronary plaque-the ZIPANGU study. Circ J. 2017;81(11):1611-9.

33. Wang J, Ai XB, Wang F, Zou YW, Li L, Yi XL. Efficacy of ezetimibe combined with atorvastatin in the treatment of carotid artery plaque in patients with type 2 diabetes mellitus complicated with coronary heart disease. Int Angiol. 2017;36(5):467-73.

34. Wang X, Zhao X, Li L, Yao H, Jiang Y, Zhang J. Effects of combination of ezetimibe and rosuvastatin on coronary artery plaque in patients with coronary heart disease. Heart Lung Circ. 2016;25(5):459-65.

35. West AM, Anderson JD, Meyer CH, Epstein FH, Wang H, Hagspiel KD, et al. The effect of ezetimibe on peripheral arterial atherosclerosis depends upon statin use at baseline. Atherosclerosis. 2011;218(1):156-62.

36. Zou YC. Effect of ezetimibe combined with low-dose atorvastatin calcium on carotid atherosclerosis in elderly patients with coronary heart disease. J Am Geriatr Soc. 2016;64(S2):S328. 